\title{
Emergence of Radial Tree of Bend Stripes in Active Nematics
}

\author{
Andrey Sokolov, ${ }^{1, *}$ Ali Mozaffari, ${ }^{2}$ Rui Zhang, ${ }^{2}$ Juan J. de Pablo, ${ }^{2,, \dagger}$ and Alexey Snezhko ${ }^{1, \hbar}$ \\ ${ }^{1}$ Materials Science Division, Argonne National Laboratory, Illinois 60439, USA \\ ${ }^{2}$ Pritzker School of Molecular Engineering, The University of Chicago, Chicago, Illinois 60637, USA \\ ${ }^{3}$ Center for Molecular Engineering, Argonne National Laboratory, Lemont, Illinois 60439, USA
}

(Received 4 December 2018; revised manuscript received 17 May 2019; published 30 July 2019)

\begin{abstract}
Living liquid crystals, a realization of active nematics where a lyotropic liquid crystal is combined with active bacteria, exhibit a plethora of out-of-equilibrium phenomena that range from active turbulence and dynamic spatiotemporal patterns to the creation and annihilation of motile topological defects. Experiments and hydrodynamic simulations are used here to report on the emergence of bend stripes, which arise as spontaneous undulations of the director field in circularly aligned lyotropic liquid crystals doped with bacteria. The interplay between bacterial-induced hydrodynamic flows and elastic forces in the material induces remarkable deformation patterns consisting of branched, radially elongated bands of a high curvature of the director field. The average number of such branches increases with the distance from the center of the circular alignment, leading to the formation of a radial tree of bands that is reminiscent of a snowflake structure. Hydrodynamic simulations, which are in agreement with the experiments, are used to explain the origin of such structures and to provide additional insights into regimes that are beyond the limit of experimental measurements. In particular, it is found that when activity is switched off in the early stages of pattern formation, a pronounced decay of bend-distortion energy ensues, with little change of the splay energy, serving to confirm that the bend stripes are an outcome of activity-driven bend-instability phenomena. Taken together, experiments and simulations demonstrate a system in which strain and geometry can be combined to dynamically manipulate pattern formation in active matter, paving the way to a deeper understanding and finer control of active colloidal systems.
\end{abstract}

DOI: 10.1103/PhysRevX.9.031014

Subject Areas: Soft Matter, Statistical Physics

\section{INTRODUCTION}

Active nematics represent a new class of nonequilibrium systems that combine the mechanical properties of liquid crystals with motility introduced on a microscopic level [1]. While the dynamics of passive nematics, molecular or colloidal liquid crystals, is well understood [2-11], the concept of "active nematics" is relatively new and brings to light intriguing phenomena [12]. One of the pioneering experimental realizations of active nematics is based on microtubule-molecular motors assays mixed with a depleting agent [13]. There, attractive depletion interactions result in a bundling of the filaments and the subsequent formation of a remarkable active gel comprised of microtubular bundles that are continuously sheared and remodeled by molecular

\footnotetext{
*sokolov@anl.gov

†depablo@uchicago.edu

‘snezhko@anl.gov
}

Published by the American Physical Society under the terms of the Creative Commons Attribution 4.0 International license. Further distribution of this work must maintain attribution to the author(s) and the published article's title, journal citation, and DOI. motors. Current examples of active nematics include microtubule-motor protein mixtures [12-15], actin-motor protein systems [16-18], bacterial suspensions [19,20], cellular nematics [21-26], and suspensions of swimming bacteria in liquid crystals (termed living liquid crystals) [27-30].

Living liquid crystals are intrinsically different from suspensions of polar, self-propelled elongated particles $[19,31,32]$ in that the nematic properties and the activity are introduced into the system through two separate subsystems. The variety of complex dynamical responses of the living liquid crystals (LLCs) originates from a tunable coupling between the long-range orientational order of the LCs and microscopically induced flows, controlled by energy input, on a particle or molecular level.

At the activity level above a certain threshold, the behavior of an active nematic resembles turbulent motion (active turbulence) [33]. It is characterized by the emergence of large-scale chaotic flows and vortices and is reported in microtubule-kinesin systems [13,34], bacterial suspensions $[19,20]$, and LLCs $[18,27]$. While in bacterial suspensions the active turbulence originates from the chaotic collective swirling motion of bacteria, turbulence in biofilament-molecular motor mixtures is driven by a continuous extension and folding of the filament bundles. 
In contrast to passive nematics, active nematics continuously create and annihilate pairs of $\pm 1 / 2$ topological defects, giving rise to nontrivial defect dynamics [29,30,35-42]. For a given level of activity, an active nematic exhibits a well-defined concentration of defects, and the effective elastic constants of the material are reduced relative to those of an equivalent passive system [17,43]. Recent experimental and computational studies of an active nematic report a system-spanning orientational order of topological defects [37]. This phenomenon has been further addressed in simulations and theoretical models $[38,39]$.

When confined, the hydrodynamic flows of active nematics can be shaped [44-48]. Active nematics confined in a $2 \mathrm{D}$ channel with a width comparable to the activityinduced length scale develop an array of equispaced vortices with opposing vorticity [49,50]. Cellular nematics confined in wide stripes [51] self-organize in a nematic phase with a director tilted at a well-defined angle with the stripe's direction and develop a shear flow at the stripe's edge due to a Fréedericksz transition driven by the activity of the cells. In 3D confined systems, recent experiments demonstrate a robust transformation of the turbulentlike dynamics of bulk active fluids into self-organized coherent macroscopic flows that persist on length scales up to meters [52]. It is shown that coherent flows form in channels with squarelike profiles and disappear in the confining channels that are too thin, suggesting that the induced flows are an intrinsically $3 \mathrm{D}$ phenomenon.

A circular confinement leads to the emergence of robust circular flow and nucleation of $\pm 1 / 2$ defects that is reported both in synthetic systems [44] and in cellular nematics [23]. By confining the active nematics into a relatively narrow circular enclosure (annuli), such patterns remain stable, even for large curvatures. In related experiments [28], where the LLC is sandwiched between two plates treated with photosensitive molecules to induce a circularly aligned pattern upon irradiation with polarized light, swimming bacteria are found to form a stable unipolar rotating swarm around the center of a spiraling vortex. In those experiments, the undulation of a bacterial trajectory is limited by a prescribed anchoring of the liquid-crystal orientation at the confining surfaces, thereby restricting the size of the resulting swarms.

In the above systems, liquid-crystal alignments are not tunable, as they are either uncontrolled or fixed by a stationary confinement. Recent experiments with radially aligned unconfined microtubule-molecular motor mixtures provide experimental access to the very first moments of the onset of instability and the subsequent transition to a turbulent state in active nematics [34]. There, the emergence of reorientational instability from an initially radially aligned state manifests by the formation of periodic concentric crimps that evolve to circular walls. Further development of the instability leads to the nucleation of defects, degradation of the orientational order, and, eventually, the turbulent regime.
In this paper, we examine the dynamics of a circularly aligned living liquid crystal in two configurations: near the bottom surface of a pendant drop attached to a glass slide and in a thin freestanding film suspended between filaments. At the beginning of our experiments, swimming bacteria and the director field of the liquid crystal are aligned concentrically by an externally controlled macroscopic hydrodynamic vortex, created by means of a ferromagnetic microbead actuated by an external rotational magnetic field. When the magnetic field is switched off, fast relaxation of the vortex due to high viscosity is observed, while the alignment remains circular. Upon full cessation of the rotation, activity-induced undulations of the LC director field start to emerge. The undulations are manifested as areas with high local curvature, which increase the free energy associated with bending deformations. Such areas appear as "branches" of a growing radial tree structure, with the number of branches increasing with the distance from the center of the system. The number of branches is controlled by the bacterial activity in the system. In fact, a bend in active systems often results in interesting instabilities $[13,19,23,27,28,34]$ and, therefore, seems to be a universal phenomenon.

Hydrodynamic simulations of the active nematic system exhibit the same branched structure observed in experiments, revealing that activity-fueled instabilities result in appreciable spatial inhomogeneities of the nematic ordering that take the form of alternating bend stripes of opposite curvature. Such bend stripes are also predicted in recent simulations and referred to as kink walls or deformation walls [53-56]. In response to the local injection of extensile active stresses, long and narrow regions of highly distorted director field are formed, which exhibit a relatively large Poiseuille-like flow along their main axis [Fig. 3(e)]. When activity is increased, a larger number of narrower bands appear and more branches are formed. Simulations also indicate that activity-driven hydrodynamic instabilities are responsible for the formation of the bands; furthermore, as soon as activity is terminated, hydrodynamic instabilities are suppressed, and the nematic orientation relaxes back to a uniform circular alignment. Interestingly, the analysis of distortion energies reveals that the bending energy declines considerably upon cessation of active stresses, whereas the splay energy remains unaltered. Taken together, concerted experiments and simulations reveal a system in which the interplay of conservative and active stresses can be used to form and control the formation of dynamic patterns. Such patterns can be manipulated by strain and geometry, thereby providing a new avenue to harness the energy of active particles [57-61] for controlled transport at the microscale.

\section{EXPERIMENTAL PROCEDURE}

\section{A. Pendant drop}

In the first series of experiments, the LLC is aligned circularly in the bottom layer of a pendant drop by a 


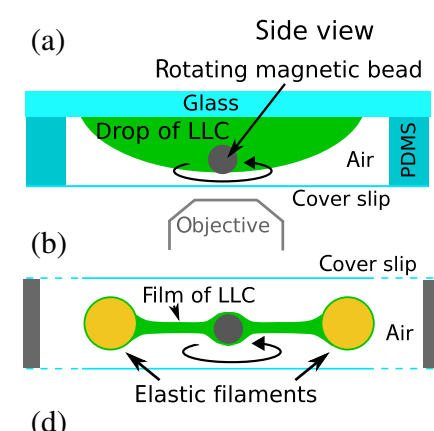

(d)
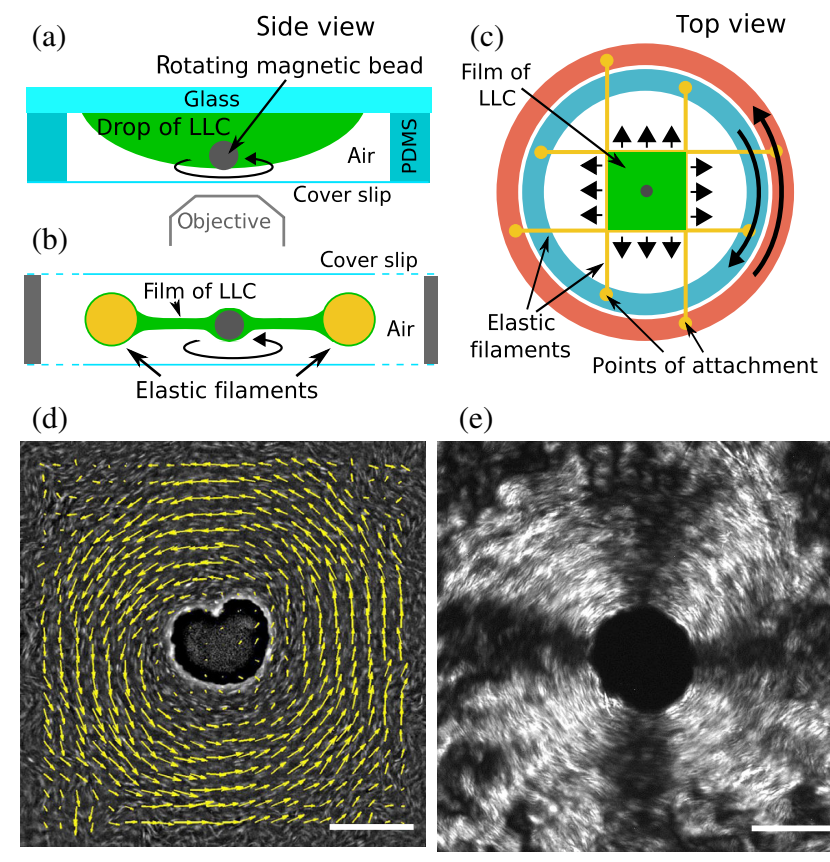

(e)

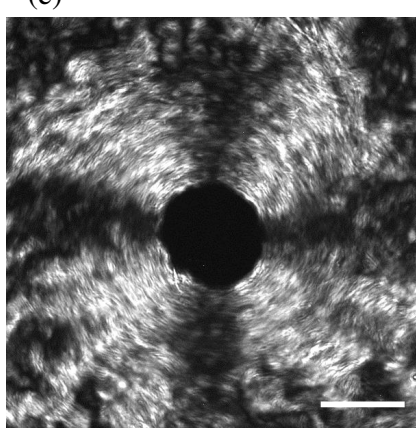

FIG. 1. (a) A sketch of the experimental setup with a pendant drop. A microscopic magnetic bead (black) is rotated by an external rotating magnetic field in a pendant drop of LLCs (green) attached to a glass slide. The drop (about $8-10 \mathrm{~mm}$ in diameter and $100-200 \mu \mathrm{m}$ thick) is enclosed to minimize evaporation. The magnetic bead is shown oversized for better representation. (b) Sketch of a side view and (c) top view of the experimental setup with an LLC film stretched between elastic filaments (yellow). The relative rotation of red and blue rings in (c) stretches the film, providing a control of the film thickness. (d) Velocity field of bacteria immediately after cessation of rotation (bottom view). The scale bar is $60 \mu \mathrm{m}$. (e) A crosspolarized microscopy image of an LC immediately after the cessation of rotation. The scale bar is $60 \mu \mathrm{m}$.

temporally induced hydrodynamic vortex; see Supplemental Movie 1 [62]. The vortex (approximately $400 \mu \mathrm{m}$ in diameter) is created by spinning a small nickel particle of radius $R=30 \mu \mathrm{m}$ with an external rotating magnetic field; see Fig. 1(a) and the Appendix A for technical details. The particle sediments at the lowest point of the pendant drop by gravity. The maximum particle rotation frequency is limited by the relatively high viscosity of the $\mathrm{LC}$ to $30 \mathrm{~Hz}$. Because of the much higher average viscosity of the LC, we do not observe a significant expulsion or concentration of bacteria in the vicinity of the particle $[63,64]$. The high anisotropy of the liquid-crystal viscosity could also contribute to the uniformity of the bacterial concentration. Nonmagnetic and nonmagnetotactic bacteria are not affected by an external magnetic field. The shear flow created by the rotating particle aligns the director in the direction parallel to the local velocity of the emerging vortex. The undisturbed director field may be approximated as $\mathbf{n}_{\mathbf{0}}=(-\sin \phi, \cos \phi, 0)$ for $r<150 \mu \mathrm{m}$, where $r$ and $\phi$ are polar coordinates. Because of a spatial decay of the vortex at large distances, the circular alignment starts to degrade for $r>150 \mu \mathrm{m}$. A "cross pattee" shape pattern is observed under cross-polarized light, with four zones having an orientation either parallel or perpendicular to the polarizer or analyzer orientation; see Fig. 1(e). The circular alignment of the director field in the vicinity of the particle while it is spinning is confirmed by rotating the polarizer-analyzer pair. After initial short relaxations of the system [Fig. 1(d)], we start tracking the bacterial velocities and orientations, as shown in Supplemental Movies 2 and 3 [62]. For relatively low bacterial concentrations (less than $5 \times 10^{9}$ cells per $\mathrm{cm}^{3}$ ), the orientations of fluorescent bacterial bodies are reliably detectable, and the local director field of the LC may be reconstructed from the local bacterial orientation (see Supplemental Movie 4 [62]), since bacterial bodies tend to align parallel to the LC director $[27,29,30]$ (see Appendix A for details).

\section{B. Freestanding 2D film}

Spinning a microbead in a pendant-drop setup efficiently aligns the LC circularly around the bead. However, since the thickness of the drop (100-200 $\mu \mathrm{m})$ is about 2-3 times larger than the bead diameter, the three-dimensionality of the flows generated by the bead and the presence of a nonslip LC-glass interface may significantly modify the dynamics of LLCs beyond a simple 2D analysis. To test the effect of the vertical drop dimension on the observable 2D LLC dynamics in the bottom layer, we perform an additional series of experiments in a thin liquid film stretched between elastic filaments; see Fig. 1(b). The elastic flexible filaments are attached to two concentric rings in a way shown in Fig. 1(c) (see Appendix A for a detailed 3D model sketch). A small drop of LLC containing a magnetic bead is stretched to the required size by contrarotating rings that move the filaments apart. This technique allows us to produce almost perfect 2D freestanding films except for the areas in the immediate vicinity of the bead and the supporting filaments. Before applying a rotating magnetic field, the magnetic bead is positioned in the film center with the help of a strongly nonuniform magnetic field created by an additional microscopic magnetic coil with a sharp ferrite core; see Fig. 8. However, the spinning particle inside the film is unstable due to the presence of surface tension forces pushing the bead towards the filaments. That makes the consistency of the initial alinement procedures problematic.

In Fig. 3, we compare the velocity relaxation rate and vorticity obtained in both setups. The velocities of bacteria are determined with a standard particle image velocimetry technique (PIV) using a custom-made MATLAB script. While the relaxation time of the vortex in a 2D freestanding film is significantly longer due to the absence of friction with walls [see Fig. 3(a)], we do not observe any noticeable difference in the development of the branched radial bend stripes with a pendant-drop geometry; see Supplemental Movie 11 [62]. Both setups by design do not develop an oxygen gradient (which could result in a gradient of activity) 
in the horizontal plane of observation in contrast to earlier experiments with LLCs [27]. Because of the higher degree of control in experiments with the pendant-drop configuration, we perform a systematic study of LLC dynamics using this setup. A vertical variation on a nematic orientation does not change qualitatively the observed behavior. A good agreement of experimental observations with 2D simulations also validates this assumption.

\section{RESULTS}

Immediately after cessation of the particle rotation, bacterial bodies align concentrically $\mathbf{n}_{\mathbf{0}}=(-\sin \phi, \cos \phi, 0)$ up to a distance of approximately $150 \mu \mathrm{m}$, while the concentration of the bacteria remains intact (see Appendix D). At larger distances $r$ (in the outer zone), the magnitude of the shear flow is insufficient to induce alignment, and the orientation of the bacteria is chaotic. In the course of vortex relaxation, bending undulations emerge in the circularly aligned LC director field; see Figs. 2(a)-2(c). Swimming bacteria create flows that decay slowly with the distance along the direction parallel to the LC orientation [65]. The hydrodynamic interaction between nearly parallel bacteria on the same streamline imposes a disorienting torque. Since the orientations of bacteria and LCs are coupled locally, this hydrodynamic torque is opposed by the elastic torque in the LCs, tending to align the bacteria back to a parallel orientation with respect to each other.

It was demonstrated previously [27] that an initially uniform orientation of the director field in the LLC $\mathbf{n}_{\mathbf{0}}=(1,0,0)$ is transformed into a striped pattern with a wavelength $\xi=\sqrt{K h /\left(\alpha_{0} l c U_{0}\right)}$, where $K$ is the average Frank elastic constant, $h$ is the vertical size of the system, $l$ is the length of a bacterium, $c$ is the local bacterial concentration, and $U_{0}$ is the strength of a bacterial hydrodynamic force dipole. Parameter $\alpha_{0} \sim 1$ is a dimensionless number that depends on the geometry of the experimental setup. The circular geometrical confinement of the current experiment, however, imposes important constraints, leading to new dynamic patterns and behavior. While bacteria swim mostly along the director field, with trajectories consisting of closed loops, the director field and bacterial trajectories start to undulate, as shown in Figs. 2(a)-2(c); also see Supplemental Movies 4 and 5 and the simulations in Supplemental Movie 6 [62]. For small perturbations, one can neglect the interaction between waves at different radii and consider independently the dynamics of the LLC in thin circular stripes. A $2 \pi$ periodicity quantizes the emerging waves and limits the possible wavelengths for each loop of radius $r$ up to $2 \pi r / n$. The number of periods $n$ is defined by the proximity of a corresponding wavelength to the favorable instability wavelength $\xi$.

The development of undulations is accompanied by an increase in radial velocities. This increase can be most clearly appreciated in simulations [Fig. 3(c)] but is also evident in experiments [Figs. 3(a) and 3(b)] (see also Supplemental Movie 7 for the emergence of vortices [Figs. 3(b) and 3(d) and Supplemental Movie 8] [62]). With time, the interactions between nematic waves become significant, leading to nontrivial dynamics. Since the number of periods depends on $r$, undulating regions of the nematic

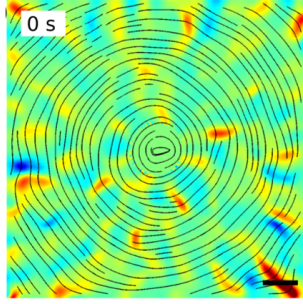

(a)

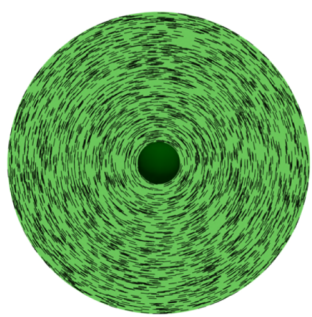

(e) $\tau^{*}=0$

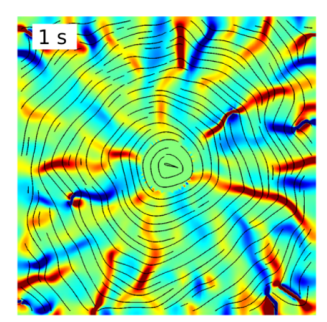

(b)

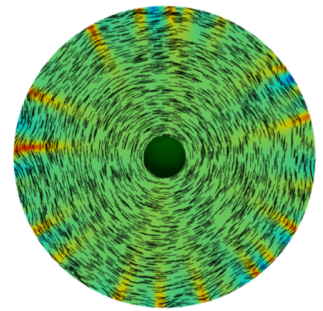

(f) $\tau^{*}=1$

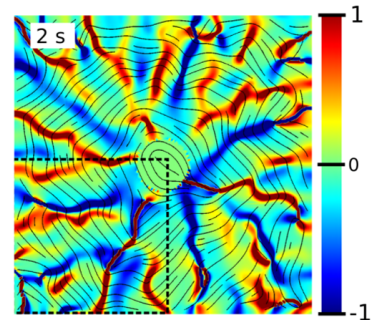

(c)

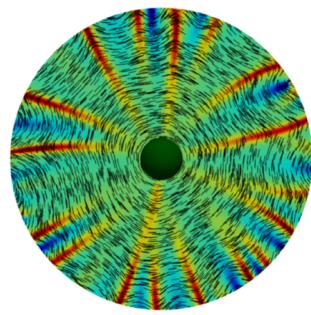

(g) $\tau^{*}=2$

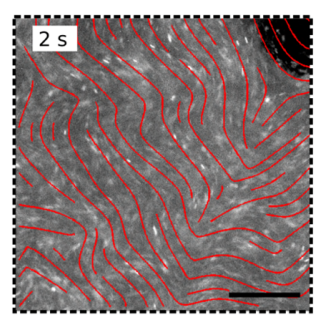

(d)

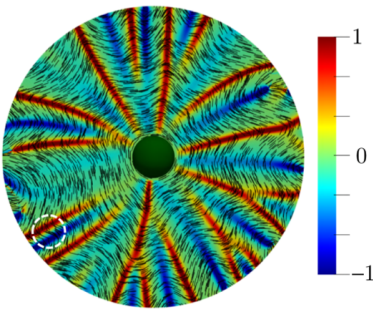

(h) $\tau^{*}=3$

FIG. 2. (a)-(c) Instability of LC orientation upon cessation of rotation. Director field and local bacterial orientations are shown with black lines. Color depicts $\kappa(r, \phi)-1 / r$, normalized by a maximum value $\left(0.015 \mu \mathrm{m}^{-1}\right)$, where $\kappa$ is a local curvature of the director field. The scale bar is $60 \mu \mathrm{m}$. (d) A cropped image of fluorescent bacteria in the areas marked by the dashed square in (c). The scale bar is $60 \mu \mathrm{m}$. (e)-(h) Simulation results for the time evolution of the director field ( $\tau^{*}$ corresponds to $10^{3}$ simulation time steps). Local curvature is mapped on the director field. Dark red (blue) represents local convex (concave) deformation, and the scale bar shows the normalized local curvature (see also Supplemental Movie 6 [62]). 


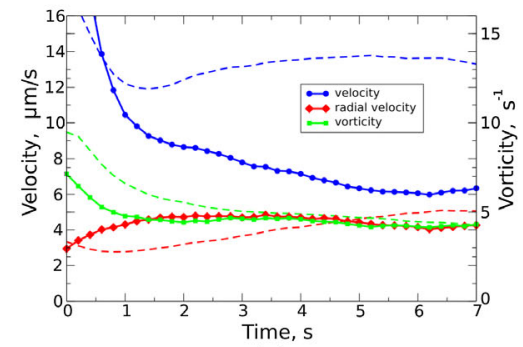

(a)

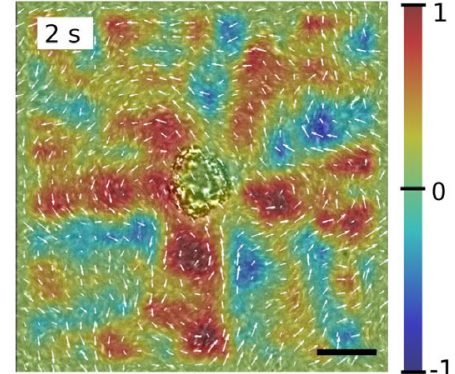

(b)

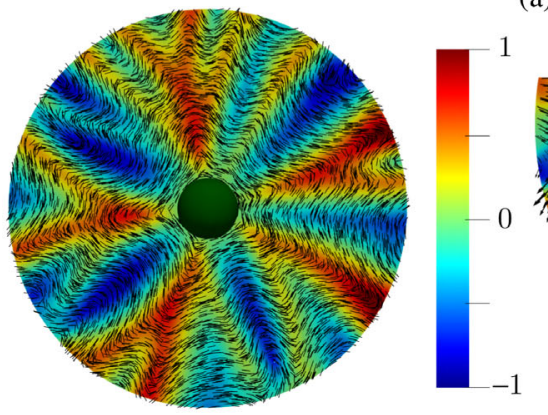

(c) $v_{r} /\left|v_{r, \max }\right|$

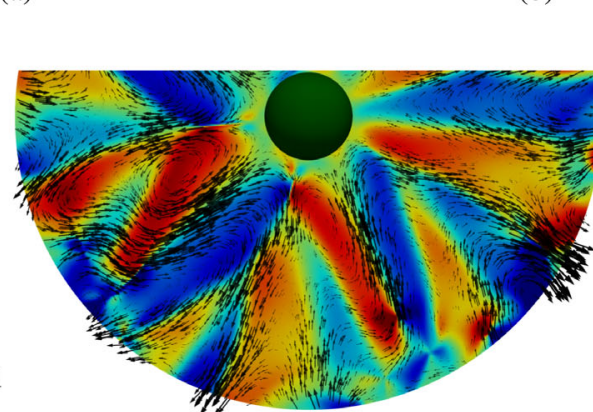

(d) $\omega /\left|\omega_{\text {max }}\right|$

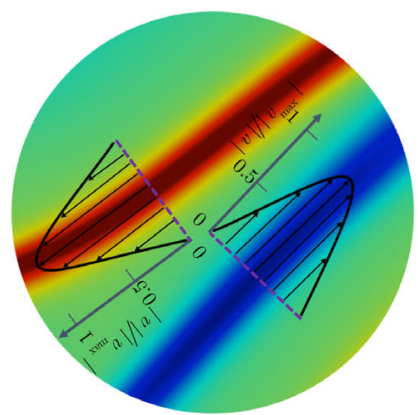

(e)

FIG. 3. (a) Relaxation of the hydrodynamic vortex and development of an active turbulence. The time evolution is averaged over the field of view. The magnitude of the velocity (blue circles), the radial component of the velocity (red diamonds), and the vorticity (green squares) are displayed. Solid lines with symbols represent results obtained in a pendant drop; dashed lines illustrate the parameters in a 2D freestanding film. (b) The vorticity map obtained from the experiment. The magnitude of the vorticity is normalized by its maximum value $\left(0.9 \mathrm{~s}^{-1}\right)$. The scale bar is $50 \mu \mathrm{m}$. (c)-(e) show results of simulations. (c) Radial velocity $\left(v_{r}\right)$ corresponding to $\tau^{*}=3$. The velocities are normalized with the maximum radial velocity of the simulation. The red regions show the regions with flows moving radially outward, and the blue ones are inward flows (see Supplemental Movie 7 [62]). (d) The vorticity map and the velocity streamlines corresponding to $\tau^{*}=3$. The vorticity $(\omega)$ magnitudes along the axis perpendicular to the plane of simulation are normalized with the highest magnitude of vorticity $\left(\omega_{\max }=1.5 \times 10^{-3}\right.$ in simulation units). The blue regions correspond to vortices with clockwise circulations, and the red are counterclockwise ones. The scale bar serves for both (c) and (d). (e) The velocity profile across two neighboring stripes of opposite curvature. The background color shows the local curvature corresponding to the dashed circular region in Fig. 2(h). The magnitude of the velocity is normalized with its maximum value along that stripe (about $10^{-2}$ in simulation units) to better present a Poiseuille-like flow along the stripes. The velocity field along the bend stripes has a parabolic shape with the highest magnitude at the center of the stripe (location of highest curvature), which quickly decays as we move across the stripe and approaches zero at the location with zero local curvature.

director field [depicted by red and blue in Fig. 2(c), depending on the sign of the curvature change] begin splitting with increasing $r$. As a result, bending and splay deformations are both present during the instability growth.

To better understand the underlying physics of the observed behavior, particularly in regimes not easily accessible to experiments, such as larger areas of the initial circular LC alignment, additional numerical simulations are performed. As a model system, we consider the standard theory of active nematodynamics to study this system $[2,31,66,67]$. Simulations are carried out in a square simulation box spanning $600 \times 600$ lattice points, with periodic boundary conditions in all directions. A no-slip boundary condition and planar anchoring are imposed on the particle surface of radius $R=25$ located at the center of the simulation box. For all simulations reported, for $|r| \leq 200$, where $r$ is the position vector of the lattice point with respect to the center of particle, the system is initialized with the director field aligned tangential to a series of concentric circles centered around the particle's centroid. Outside this region, the director has a random orientation within the plane of the system. We use a hybrid lattice Boltzmann-finite difference method to solve the active nematohydrodynamics equations [67] (refer to Appendix B for more details).

The interplay of activity-induced spontaneous flow that drives the system away from the minimum of the free energy, and the orientational ordering of the liquid crystal, gives rise to a rich dynamical behavior over length scales much larger than the size of each individual active entity. The effect of hydrodynamic flows generated by the forcefree swimming bacteria is considered by adding an extensile active stress to the equations of motion, which accounts for continual energy injection at the microscale (see Appendix B for details). Results for the time evolution of the director field and the local curvature are compared to experiments in Fig. 2. In agreement with experimental observations, Figs. 2(e)-2(h) show that the extensile 


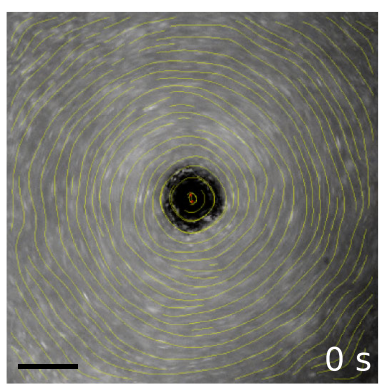

(a)

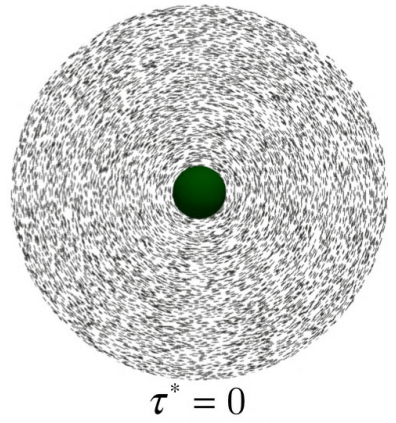

(d)

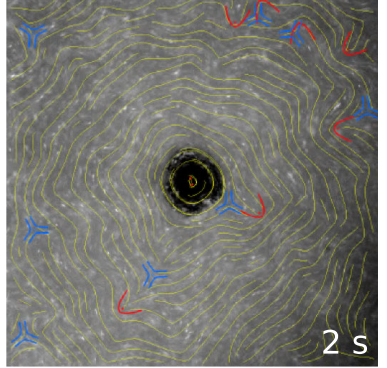

(b)

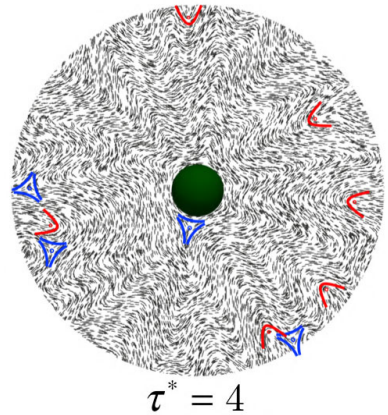

(e)

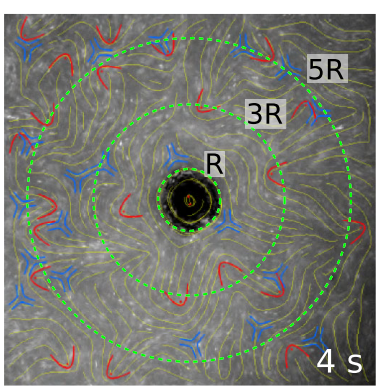

(c)

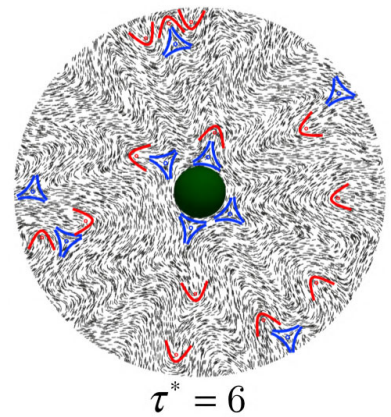

(f)

FIG. 4. The emergence of topological defects in a circularly aligned LLC as observed in experiments (a)-(c) and simulations (d)-(f). $+1 / 2$ defects are depicted by red; $-1 / 2$ defects are depicted by blue. Dashed green lines outline two areas used for defect statistics analysis in Fig. 5. The scale bar is $60 \mu \mathrm{m}$.

nature of the active stress induces bending instabilities with a spontaneous distortion of the director field. In experiments, the bending instability develops first for relatively large radii $r$ and then propagates towards the center [Figs. 2(a)-2(c) and Supplemental Movie 5 [62] ]. The same behavior is recovered in simulations (see Supplemental Movie 6 [62]). Hydrodynamic instabilities originating from the active stress destabilize the nematic ordering by localizing the deformations over narrow regions, to form undulations that propagate radially towards the particle, and generate bend stripes of alternating curvature. Highly distorted bands are accompanied by the lower scalar order parameter, due to the destruction of long-range orientational ordering of the nematic and the longitudinal flow along them, which is radial towards the particle for concave deformations (distinguished by the blue in the curvature maps) and away for convex deformations (red stripes); see Fig. 3(c). As shown in Fig. 3(d), the bend stripes form at the interface of vortices of opposite circulation. At later times, the director undergoes additional distortions, and the system finds it more energetically favorable to unbind pairs of $\pm 1 / 2$ defects. The newly created $+1 / 2$ defect glides through the band to reduce the elastic distortion energy and also relax the high shear stress localized at the bands. Simulations also reveal that the $+1 / 2$ defects, which are self-propelled and in the extensile systems move toward their head, can destabilize the nematic ordering within the region near the particle to form concave deformations. The region in between the two $+1 / 2$ defects deforms to create convex deformations. This deformation further increases the bending deformations and contributes to the formation of new undulations. The emergence of topological defects in a typical experiment and simulations is shown in Fig. 4 (Appendix C).

Observed instability patterns with radially elongated stripes introduce a strong anisotropy to the angular distribution of the emerging topological defects; see Fig. 5. In the beginning, the $+1 / 2$ defects nucleate with their orientations close to $\varphi=0$ and $\varphi=\pi$ (along radial directions), while $-1 / 2$ defect orientations are localized along $\varphi=0$. Over time, a transition to a chaotic motion of defects results in the spreading of defects' orientations. The bending instability develops first at large distances $r$ [inset in Fig. 6(b)]. As a result, the proliferation of the emerging defects is observed earlier for large distances, $3 R<r<5 R$, when compared to the short distances, $R<r<3 R$; see Figs. 5(c) and 5(d) and Figs. 5(a) and 5(b). The simulations also show that the self-propelling $+1 / 2$ defects which point towards the ordered region $(\phi=\pi)$ first enter from the chaotic region [see Figs. 5(e) and 5(g)].

The injection of energy into the system is facilitated by the consumption of oxygen and nutrients from the medium by the bacteria and the subsequent conversion of the energy into hydrodynamic flows generated by each bacterium. The activity in the system is controlled by both the concentration and average speed of the bacteria that depend on the level of oxygen in the media. 


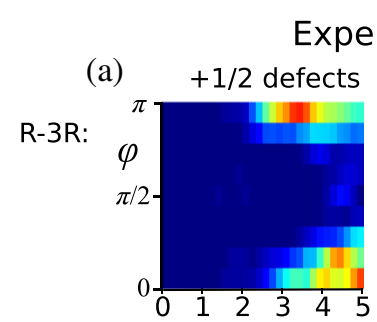

Experiments:

(c)

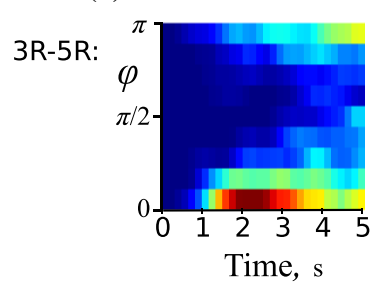

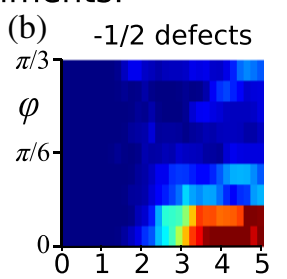

(d)

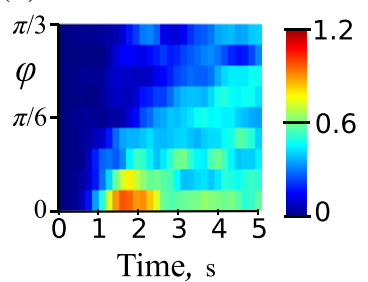

Simulations:
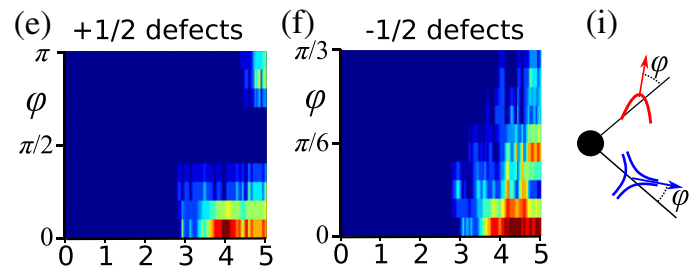

(g)

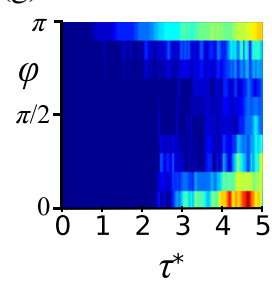

(h)

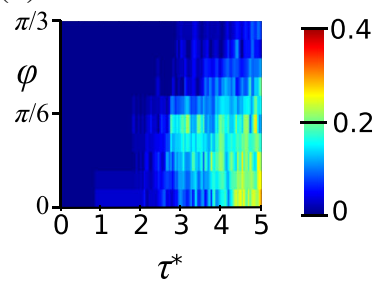

FIG. 5. The temporal evolution of defect orientation statistics in experiments (a)-(d) and simulations (e)-(h). Color represents a number of $+1 / 2$ defects (a),(c),(e),(g) and $-1 / 2$ defects (b),(d),(f),(h) with orientation $\varphi$ per area $R^{2}=900 \mu \mathrm{m}^{2}$ at a given time. The temporal evolution is observed in the inner area-between $r=R$ and $r=3 R$ (a),(b),(e),(f) (top row)—and the outer area-between $r=3 R$ and $r=5 R(\mathrm{c}),(\mathrm{d}),(\mathrm{g}),(\mathrm{h})$ (bottom row). For illustration purposes, these lines are shown as dashed green lines in Fig. 4(c). The orientation of defects is defined in (i) and is assumed to be symmetrical relative to $\varphi=0$, and only the positive part is shown. Seven experiments with activity $\zeta_{1}$ are processed for (a)-(d). The scale bar in the simulations shows the defect density defined as the number of defects per unit area of interest. The statistics presented correspond to the average of ten simulation runs.

To characterize and change the activity level in the experiments, we use activity parameter $\zeta \sim c V$, where $c$ and $V$ are the concentration of the bacteria and average bacterium speed, respectively.

In order to examine how changes in activity lead to the development of dynamic patterns presented here, we perform a series of experiments with five bacterial suspensions at different activity levels $\zeta$. We recreate the vortex four times on the same bacterial suspension, analyze the development of the bending instability, and present the results averaged over four experimental realizations for each of the five prepared bacterial suspensions.

The instability growth may be characterized by an azimuthally averaged peak-to-peak nematic deflection angle $\theta_{p p}(r, t)=\left\langle|[\theta(r, \phi, t)-\phi-\pi / 2]|_{p p}\right\rangle_{\phi}$, where $\theta(r, \phi, t)$ is the local nematic orientation angle. Evolution of the deflection angles, $\theta_{p p}(r, t)$, as a function of the distance from the particle, at different times [shown as an inset in Fig. 6(b)] reveals that the instability develops first at large $r$ and then propagates inside. A time evolution of the $\theta_{p p}$ averaged over the field of view for different bacterial activities $\zeta$ is presented in Fig. 6(b).

The experimental system undergoes a transition to an active turbulent state $[13,27,33]$ when $\left\langle\theta_{p p}\right\rangle_{r}$ exceeds a value of approximately 2 . For each $\zeta$, we also measure the number of undulations (petals) as a function of distance $r$ at the intermediate state of the instability $\left(\theta_{p p} \approx 1 \mathrm{rad}\right)$. The number of undulations at a fixed $r$ increases with activity $\zeta$; see Fig. 6(a). Correspondingly, the instability wavelength averaged over the field of view decreases with activity, since $\xi \sim \sqrt{\zeta_{1} / \zeta}$; see Fig. 6(c).
The effect of activity strength on the formation of bending instabilities is clearly demonstrated in simulations. The suspension of extensile active units is inherently unstable to the bending deformation, as it is shown that bending perturbations grow exponentially with time $[31,56,68,69]$. The critical activity strength that leads to a transition from quiescent to spontaneous flow scales as $1 / h^{2}$ [68], where $h$ is the confinement width (see Ref. [51] for the experimental observation of spontaneous development of shear flow in a cellular nematic system). Two-dimensional unbounded systems, therefore, show hydrodynamic instabilities even at the slightest activity input. The results shown in Fig. 6(e) and similar experimental observations displayed in Fig. 6(a) illustrate that the number of undulations increases with the activity strength and confirm that a larger number of narrower bend stripes are created [also see Figs. 6(g) and $6(\mathrm{~h})$ ]. While the experimental system is quasi-2D due to the pendant-drop geometry of the experiment, the observed dynamics of the undulations are still consistent with the simulation results. For a given value of the activity strength, the width of undulations remains fairly constant [see Fig. 6(h)], indicating that the size of the petals is insensitive to the change in the curvature. This result necessarily leads to the formation of more branches at larger distances. The time evolution of the mean deflection angle, which represents the magnitude of the deviation of the director from the initial circular alignment, is summarized in Fig. 6(f). At higher activity strengths, the local energy input is enhanced, leading to more pronounced spatial inhomogeneities in the director field over a shorter period of time; the dynamics of instability initiation and growth therefore 


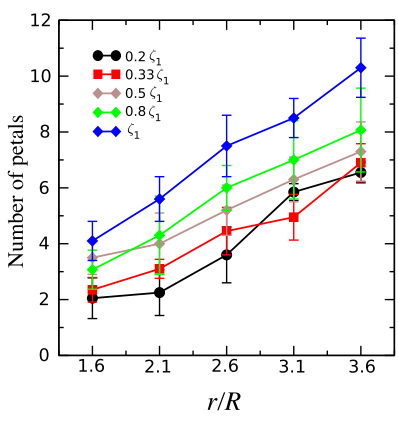

(a)

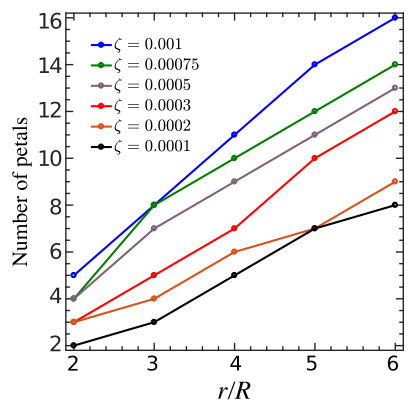

(e)

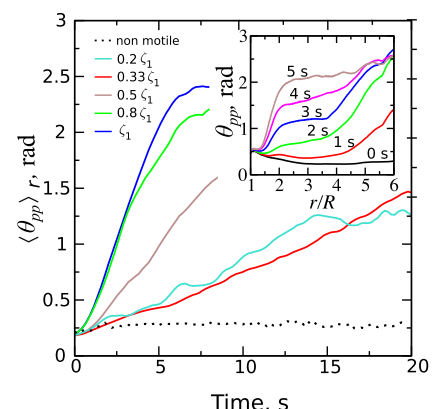

(b)

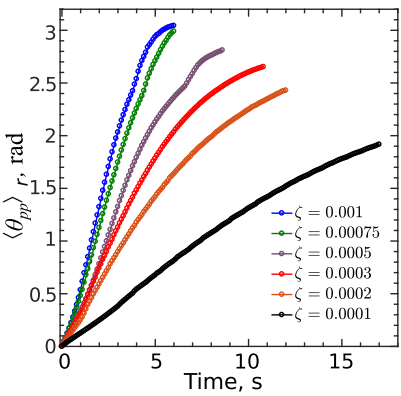

(f)

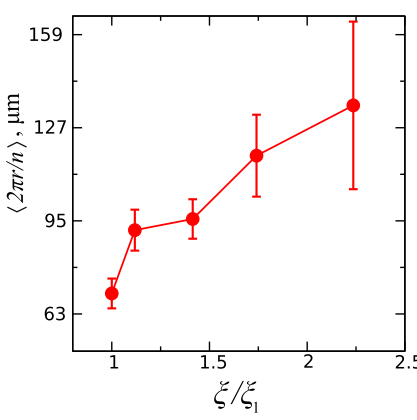

(c)

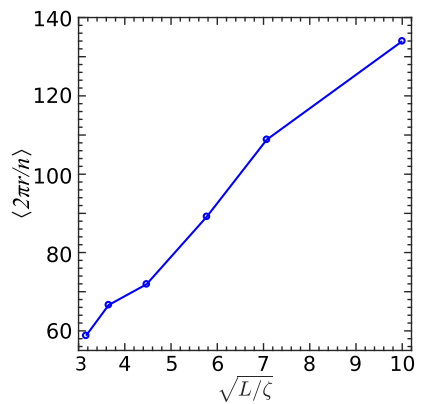

(g)

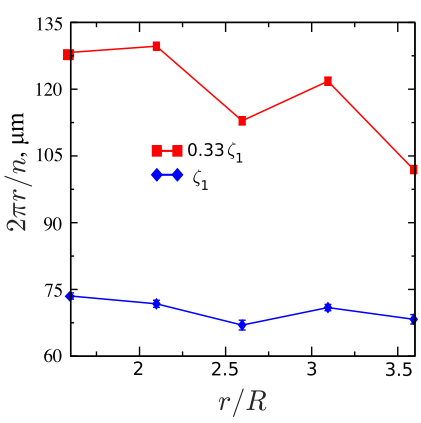

(d)

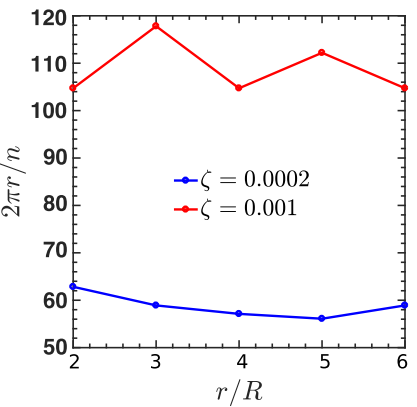

(h)

FIG. 6. (a) A number of positive curvature undulations (petals) as a function of distance $r$ from the center of the circular alignment for different bacterial activities $\zeta$. (b) The temporal evolution of the peak-to-peak nematic deflection angle $\theta_{p p}$ averaged over the region of interest. Inset: $\theta_{p p}(r, t)$ as a function of $r$ for different moments of time after the cessation of the particle rotation. Four experiments with the same particle and bacterial activity contribute to the statistics. (c) Average wavelength $\langle 2 \pi R / n\rangle$ of bending instability as a function of the normalized activity-induced length scale, $\xi_{1}=75 \pm 20 \mu \mathrm{m}$. (d) The wavelength of bending deformations as a function of the dimensionless radial distance from the particle center for two values of activity strength. (e)-(h) The corresponding simulation results; see Appendix B for the explanation of the simulation parameters.

become faster. The medium dynamic viscosity $(\eta)$ damps the local energy input, so the timescale at which the active stress is injected into the system is proportional to $\sim \eta / \zeta$, and the nematic LC's response rate which relaxes the distortion is proportional to $l^{2} /(\Gamma L)$, where $L, \Gamma$, and $\zeta$ are the elastic constant, rotational diffusivity, and activity strength, respectively. The dynamic of bending instabilities formed in the system is determined by the interplay of these two timescales. By increasing the activity strength, the relaxation of the nematic to a uniform state lags behind the spontaneous distortion of the director induced by the local energy input, leading to a higher instability growth rate, as depicted in Fig. 6(f). In Fig. 6(g), we report the variation of $2 \pi\langle R / n\rangle$, which represents the average width of bending deformation as a function of induced activity length scale $\sqrt{L / \zeta}$, over which active $\Pi^{a}$ and passive $\Pi^{p}$ stresses are balanced. The interplay between autonomously generated hydrodynamic flows, which disturb the director field, and the elastic forces, which resist that disturbance, determines the length scale over which the active stress is accommodated by the elastic restoring forces. As $\sqrt{L / \zeta}$ decreases, the liquid-crystal microstructure can no longer accommodate the active stress, and the characteristic size of the vortices therefore decreases (in experiments, a characteristic size of the observed vortices varies from 30 to $60 \mu \mathrm{m}$ ). Vortices become faster, and the system shows a larger number of short-lived and narrower bend stripes. These results are in agreement with the experiments and explain the characteristic properties that emerge in our system.

In order to gain additional insights into the activitydriven hydrodynamic instability reported here, we turn our attention to simulations in which active stress is switched off as soon as the bending deformations appear in the system. Figures 7(a)-7(c) show a series of snapshots of the director field, with the corresponding local curvature highlighted, for a system in which activity is turned off at $\tau^{*}=2$ [see Fig. 2(g)]. One can appreciate that active stresses are indeed responsible for the formation of undulations and narrow regions of high bending deformations. After cessation of activity, the director field relaxes back to an undulation-free configuration, and the circular alignment is restored as a result of the high elastic forces stored in the bands. The change of total splay $(\boldsymbol{\nabla} \cdot \mathbf{n})^{2}$ and bend $[\mathbf{n} \times(\boldsymbol{\nabla} \times \mathbf{n})]^{2}$ distortion energy shows the dominance of bending deformations over splay deformations, particularly in the early stages of instability formation [Fig. 7(e)]. Note that both bend and splay energies increase as the bands undergo further deformations as a result of highly localized flows within the bands, which cause a higher growth rate of 


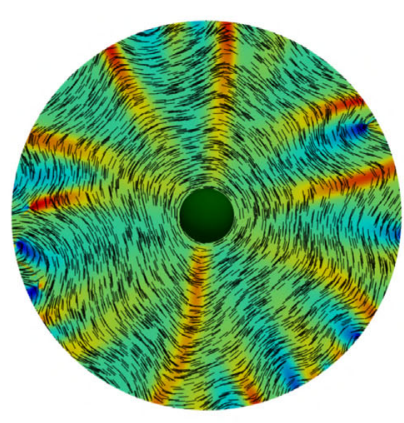

(a) $\tau^{*}=4$

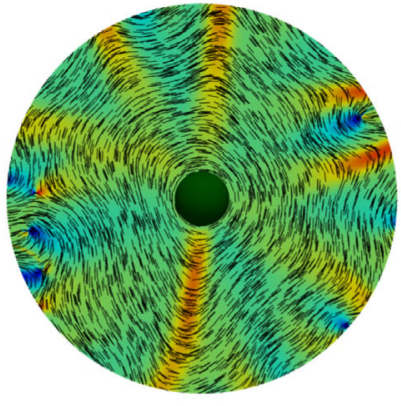

(b) $\tau^{*}=8$

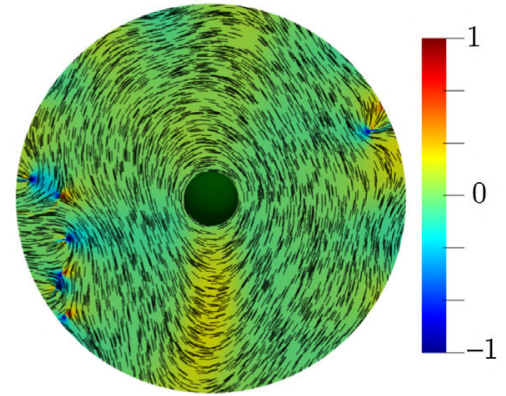

(c) $\tau^{*}=40$

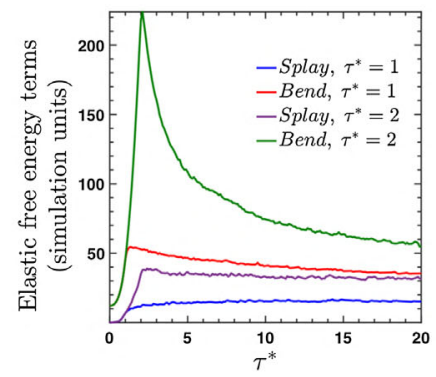

(d)

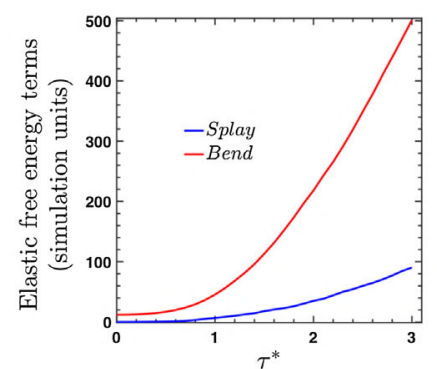

(e)

FIG. 7. (a)-(c) Simulation results for the time evolution of the director field. Color depicts $\kappa(r, \phi)-1 / r$, normalized to a maximum value, where $\kappa$ is the local curvature of the director field. The activity is turned off at $\tau^{*}=2$ [see Fig. 2(g)]. Local curvature is mapped on the director field. Dark red (blue) represents local convex (concave) deformation (see Supplemental Movie 9 [62]). The variation of the total bend and splay in the system as a function of the time for a system with (d) activity turned off at $\tau^{*}=1$ [see Fig. 2(f)] and $\tau^{*}=2$ [see Fig. 2(g)] and (e) constant activity as depicted in Figs. 2(e)-2(h).

the splay term at a later time in order to reduce the high shear stress and deformations in the bands. As soon as activity is turned off, bend deformations decay abruptly. The magnitude and the rate of decay are higher for the system in which activity stops at a later time [Fig. 7(d)], underscoring the high bending deformations that arise as a spontaneous response of the director field to the local injection of the extensile dipolar force. The splay energy remains fairly constant throughout the simulation and decays at much later times as defect pairs annihilate each other. These results are simply a manifestation of the high elastic force that is stored in the bend stripes. Interestingly, the bending energy has an elasticlike response to the injection and cessation of the active stress, whereas the splay deformations behave in a more "plastic" manner. We note here that the recovery to the circular alignment could be enhanced by increasing the anchoring strength at the particle surface.

It is of interest to confirm that elastic stresses accumulated in the LC over the course of particle rotation do not contribute to the development of undulations. Additional experiments with nonmotile bacteria uniformly distributed over the drop are performed to address this concern. After stopping the particle rotation, the nematic orientation and bacterial bodies remain circularly aligned with no undulations; see Supplemental Movie 10 [62]. The initial fluctuations of the director orientation do not grow in time; see Fig. 6(b). These results confirm that the interplay between activity and elasticity are key to the emergence of the observed branched patterns. Bend stripes are driven by activity and rapidly decay when the active system transitions back into a passive state. In addition, the long time behavior observed in simulations shows that the system evolves towards chaotic dynamics, with the continuous creation and annihilation of defects and the formation and splitting of bend stripes, particularly for higher activities. Some of the $-1 / 2$ defects are attracted to the particle surface due to the elastic forces, whereas the self-propelled, cometlike $+1 / 2$ defects swim in the medium.

\section{CONCLUSION}

Experiments and simulations have been used to investigate the creation of radial trees of bend stripes in circularly aligned active liquid crystals consisting of a nontoxic lyotropic liquid crystal doped with live bacteria. The interplay between bacteria-induced hydrodynamic flows and the elasticity of the circularly aligned liquid crystal leads to the onset of intriguing dynamic patterns, consisting of branched, radially elongated bands of high curvature. These dynamic bend stripes are accompanied by strong radial hydrodynamic flows, which could potentially be used to direct transport at the microscale. Simulations are in good agreement with experiments and help explain 
the origin and magnitude of the structures reported here. As undulations grow, the bend energy increases much faster than the splay energy. Upon switching off the activity in the early stages of pattern formation, the undulations relax as the bend drops rapidly, with the splay barely changing, indicating that the bend stripe pattern is driven by activity and the system stores energy preferably in the form of bend distortions. As such, we have demonstrated an active matter system in which the interplay of activity, elasticity, and geometry leads to a well-controlled pattern formation, paving the way towards finer control of dynamic structures and transport phenomena in out-of-equilibrium materials.

\section{ACKNOWLEDGMENTS}

The research was supported by the U.S. Department of Energy, Office of Science, Basic Energy Sciences, Materials Sciences and Engineering Division. The development of software for hydrodynamic simulations of liquid crystals was supported by the Midwest Integrated Center for Computational Materials (MICCoM), funded by the Department of Energy, Basic Energy Sciences, Materials Sciences and Engineering Division.

A. S., A. M., and R. Z. contributed equally to this work.

\section{APPENDIX A: EXPERIMENTAL METHODS AND MATERIALS}

\section{Bacteria preparation}

We use nonmagnetotactic Bacillus subtilis fluorescent strains DK400 (mCherry) $(0.8 \mu \mathrm{m}$ in diameter and 5-7 $\mu \mathrm{m}$ in length) [70] in our experiments. The bacteria are initially grown on an LB (lysogeny broth) agar plate at room temperature (approximately $22^{\circ} \mathrm{C}$ ) and then transferred to a TB (terrific broth) liquid medium with IPTG (isopropyl $\beta-D-1$ thiogalactopyranoside) at a concentration of $1 \mathrm{mM}$. Bacteria are grown in a shaking incubator at a temperature of $30^{\circ} \mathrm{C}$ for $10-15 \mathrm{hr}$ until the end of their exponential growth state, while the density is monitored by measuring optical opacity. The bacteria are concentrated by centrifugation to achieve the required density. Concentrated bacteria are transferred to a mixture of disodium cromoglycate (DSCG) and terrific broth liquid medium at an initial 20 wt. \% concentration, prepared by the protocol below. For control experiments, bacteria are killed by transferring them into a glass container and heating to $90{ }^{\circ} \mathrm{C}$ and then cooling down to room temperature. Dead bacteria are transferred to a DSCG-TB mixture in the same way.

\section{Living liquid crystal preparation}

An LLC is prepared in two steps. First, we dissolve disodium cromoglycate salt (DSCG from Spectrum Chemicals) in TB (from Sigma Aldrich) liquid medium to achieve the concentration of $20 \%$ salt by weight. The solution is heated to $60-70{ }^{\circ} \mathrm{C}$ and mixed with a vortex mixer for a minute for full dissolution of the salt. Second, the concentrated bacterial suspension is added to this solution such that the final concentration of DSCG is approximately $12 \%$ by weight. The physical properties of a DSCG-based liquid crystal strongly depend on the DSCG concentration and temperature [71]. The bacterial volume fraction is approximately $0.5 \%$. The liquid crystallinity of the prepared solution is verified by a cross-polarized microscopy via the presence of typical Schlieren textures.

\section{Experimental procedure}

A nickel magnetized particle of radius $R \approx 30 \mu \mathrm{m}$ is placed in the center of a $25 \mathrm{~mm} \times 25 \mathrm{~mm}$ microscope slide. A small drop of LLC $(5 \mu L)$ is pipetted over the particle and then enclosed by a small round rubber washer and an antifog covered glass coverslip. This full enclosure maintains the humidity level close to $100 \%$ in the chamber and noticeably reduces evaporation of even a small fraction of water from the lyotropic liquid crystal, preventing a change of viscosity and elasticity of the LLC in the drop. When the microscope slide is inverted upside down, the nickel particle sinks to the bottom surface of the pendant drop and stays in the center due to gravitational depression of the surface. During experiments, the nickel particle is rotated by an external rotational magnetic field created by two perpendicular pairs of magnetic coils.

\section{Microscopy and image processing}

We use an inverted microscope Olympus IX71 with a computer-controlled motorized stage mounted on a piezoelectric isolation platform Herzan TS-150. Sequences of fluorescent images of LLC temporal dynamics are recorded by a monochrome Prosilica GX 1660 camera $(1600 \times 1200)$ with the frame rate of $10 \mathrm{fps}$, at $10 \times$ magnification. The acquired images are processed in MATLAB with a custom script. The nematic director orientation of the LC is reconstructed from the local orientation of the bacteria. The orientations of the bacteria are estimated using the gradient method. Since a bacterial body is elongated, the local gradient of fluorescent brightness achieves a maximum value in the direction perpendicular to the bacterial orientation, except for pixels in the proximity of bacterial heads and tails. The velocity field of bacterial motion is calculated by a custom PIV script in MATLAB. The subwindows for PIV are $15 \mu \mathrm{m} \times$ $15 \mu \mathrm{m}$ downsized to $7 \mu \mathrm{m} \times 7 \mu \mathrm{m}$ for the second pass for a better spatial resolution.

\section{Freestanding film experimental setup}

The design of the experimental setup shown in Fig. 8 is adopted from previous experiments [72]. In this work, we significantly modify the experimental cell to minimize evaporation of water from the freestanding film by reducing 


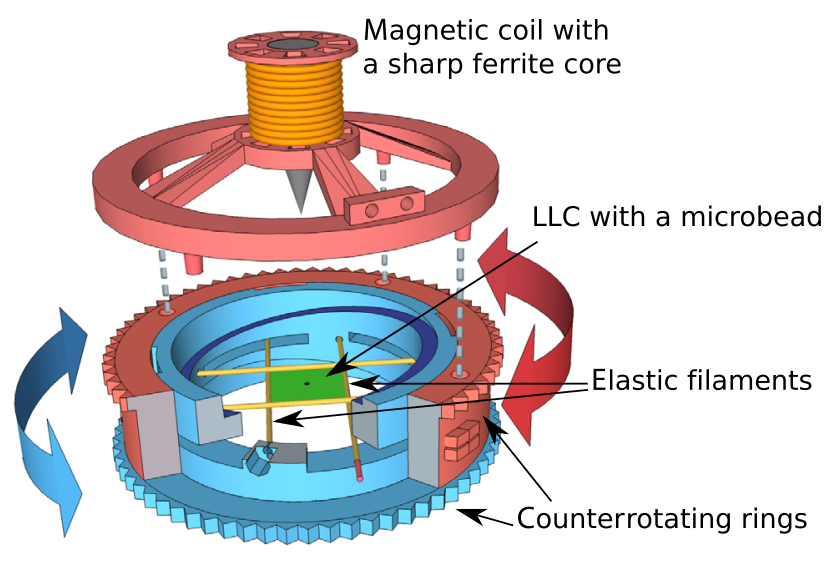

FIG. 8. A 3D model of the freestanding film experimental setup. A drop of LLC with a magnetic bead is placed between four movable elastic filaments. The design allows elastic filaments to travel freely with the rotation of the external (red) ring with respect to the internal one (blue). Counterrotation of the rings changes the area of the film between four filaments, thus adjusting the film thickness. The film is enclosed by two round glass slides mounted inside the rings to suppress evaporation. The position of the magnetic bead in the film is controlled by a nonuniform magnetic field created by a magnetic coil with a sharp ferrite core.

the volume of enclosure and designing the stretching mechanism around it. The volume of the enclosed cylindrical chamber (26 $\mathrm{mm}$ in diameter and $2 \mathrm{~mm}$ thick) is decreased to approximately $10^{3} \mu \mathrm{L}$, almost eliminating evaporation of water from a $10 \mu \mathrm{L}$ enclosed liquid film. Glass slides enclosing the cell are installed slightly unparalleled to eliminate multiple reflections. Surface tension and gravitational forces acting on the spherical particle in a saddle-shape film extrude the nickel particle from the center of the film. To minimize this effect, we create an attracting magnetic force with a small magnetic coil positioned over the center of the film.

\section{APPENDIX B: NUMERICAL SIMULATIONS}

The theory of active nematodynamics is employed to account for spatiotemporal evolution of a coarse-grained orientational order parameter $\mathbf{Q}$, a symmetric, traceless, second rank tensor. For uniaxial systems, it can be written in the form $\mathbf{Q}=S(\mathbf{n n}-\mathbf{I} / 3)$, where $\mathbf{n}$ is the nematic director field and $S$ denotes the magnitude of orientational ordering ( $3 / 2$ times the magnitude of the largest eigenvalue of $\mathbf{Q}$ ). The evolution of this nonconserved order parameter follows the Beris-Edwards equation [10]:

$$
\left(\frac{\partial}{\partial t}+\mathbf{u} \cdot \boldsymbol{\nabla}\right) \mathbf{Q}-\mathbf{S}=\Gamma \mathbf{H}
$$

The first term on the left-hand side is the material derivative which describes the rate of change of $\mathbf{Q}$ advected by the flow field. The advection term is generalized by the tensor $\mathbf{S}(\mathbf{Q}, \boldsymbol{\nabla u})$, which accounts for the response of the nematic order parameter to the symmetric $\mathbf{A}$, and antisymmetric $\boldsymbol{\Omega}$, parts of the velocity gradient tensor $(\nabla \mathbf{u})$.

The molecular field $\mathbf{H}$ embodies the relaxational dynamics of the nematic and obtained from the variational derivative of the free energy given by

$$
\mathbf{H}=-\left(\frac{\delta \mathcal{F}}{\delta \mathbf{Q}}-\frac{\mathbf{I}}{3} \operatorname{Tr} \frac{\delta \mathcal{F}}{\delta \mathbf{Q}}\right)
$$

which diffusively drives the system toward the configuration with the minimum of free energy at the rate given by the rotational diffusion constant $\Gamma$. The equilibrium thermodynamic properties of the nematic liquid crystal are determined by Landau-de Gennes free energy density $f$, which is comprised of a bulk term and an elastic term [2]:

$$
\begin{aligned}
f= & \frac{A_{0}}{2}\left(1-\frac{U}{3}\right) \operatorname{Tr}\left(\mathbf{Q}^{2}\right)-\frac{A_{0} U}{3} \operatorname{Tr}\left(\mathbf{Q}^{3}\right)+\frac{A_{0} U}{4}\left[\operatorname{Tr}\left(\mathbf{Q}^{2}\right)\right]^{2} \\
& +\frac{L}{2}(\mathbf{\nabla} \mathbf{Q})^{2} .
\end{aligned}
$$

The phenomenological coefficient $A_{0}$ sets the energy scale, and the parameter $U$ controls the magnitude of the order parameter through $S=0.25+0.75 \sqrt{1-8 /(3 U)}$.

The local fluid density and velocity are governed by the generalized incompressible Navier-Stokes equations

$$
\begin{gathered}
\boldsymbol{\nabla} \cdot \mathbf{u}=0, \\
\rho\left(\frac{\partial}{\partial t}+\mathbf{u} \cdot \boldsymbol{\nabla}\right) \mathbf{u}=\boldsymbol{\nabla} \cdot \boldsymbol{\Pi} .
\end{gathered}
$$

The total asymmetric stress tensor $\boldsymbol{\Pi}=\boldsymbol{\Pi}^{p}+\boldsymbol{\Pi}^{a}$ in the above equation is a sum of a passive and an active stress. The force free constituent active particles produce active mechanical stresses with far-field flow approximated by the force dipole which after coarse-graining give rise to the active stress of the form $[31,56]$

$$
\boldsymbol{\Pi}^{a}=-\zeta \mathbf{Q}
$$

where $\zeta$ is the measure of activity strength that generates flow when $\mathbf{Q}$ has a spatial gradient with $\zeta>0$ for extensile and $\zeta<0$ for contractile systems. For a complete description of the model and the numerical details, please refer to model details [62]. 


\section{Model details}

$\mathbf{S}$ takes the following form:

$$
\begin{aligned}
\mathbf{S}= & (\xi \mathbf{A}+\mathbf{\Omega}) \cdot\left(\mathbf{Q}+\frac{\mathbf{I}}{3}\right)+\left(\mathbf{Q}+\frac{\mathbf{I}}{3}\right) \cdot(\xi \mathbf{A}-\mathbf{\Omega}) \\
& -2 \xi\left(\mathbf{Q}+\frac{\mathbf{I}}{3}\right)(\mathbf{Q}: \nabla \mathbf{u}) .
\end{aligned}
$$

The alignment parameter $\xi$ is related to the material properties (e.g., aspect ratio of mesogens) of the liquid crystal and controls whether the director field is flow aligning or flow tumbling under a simple shear flow.

At the boundary surface with unit normal $\nu$, the anchoring condition is imposed by adding a surface term to the free energy. The fourth-order Fournier-Galatola free energy density is adopted to apply the degenerate planar anchoring boundary condition [73]

$$
f_{s}=\frac{1}{2} \mathcal{W}\left(\overline{\mathbf{Q}}-\overline{\mathbf{Q}_{\perp}}\right)^{2}+\frac{1}{4} \mathcal{W}\left(\overline{\mathbf{Q}}: \overline{\mathbf{Q}}-S^{2}\right)^{2},
$$

where $\mathcal{W}$ controls the anchoring strength, $\overline{\mathbf{Q}}=\mathbf{Q}+\frac{1}{3} S \boldsymbol{\delta}$, its projection to the surface $\overline{\mathbf{Q}_{\perp}}=\mathbf{p} \cdot \overline{\mathbf{Q}} \cdot \mathbf{p}$, and $\mathbf{p}=\boldsymbol{\delta}-\boldsymbol{\nu} \nu$.

Passive stress is defined as

$$
\begin{aligned}
\boldsymbol{\Pi}^{p}= & 2 \eta \mathbf{A}-P_{0} \mathbf{I}+2 \xi\left(\mathbf{Q}+\frac{\mathbf{I}}{3}\right)(\mathbf{Q}: \mathbf{H})-\xi \mathbf{H} \cdot\left(\mathbf{Q}+\frac{\mathbf{I}}{3}\right) \\
& -\xi\left(\mathbf{Q}+\frac{\mathbf{I}}{3}\right) \cdot \mathbf{H}-\mathbf{\nabla} \mathbf{Q}: \frac{\delta f}{\delta \boldsymbol{\nabla} \mathbf{Q}}+\mathbf{Q} \cdot \mathbf{H}-\mathbf{H} \cdot \mathbf{Q},
\end{aligned}
$$

where $\eta$ is the isotropic viscosity and $P_{0}$ is the isotropic bulk pressure. The viscoelastic properties of the nematic liquid crystal are lumped in the passive stress, which is the sum of viscous [the first term in the rhs of Eq. (B9)] and elastic terms.

Simulations are performed on a $600 \times 600$ twodimensional lattice with periodic boundary conditions in both directions. The medium viscosity is set to $\eta=$ $1 / 6$, and the liquid crystal rotational viscosity $\Gamma=0.3$. We choose the following parameters throughout the simulation: $A_{0}=0.3, L=0.01, U=3.0$ (giving $S=0.5$ ), and $\xi=0.7$ corresponding to the flow aligning liquid crystal, planar anchoring imposed on the particle surface by setting $\mathcal{W}=0.01$, and the particle radius $R=25$. We employ the hybrid lattice Boltzmann method to solve coupled governing partial differential equations [Eqs. (B1), (B4), and (B5)]. The time integration is performed using the Euler forward scheme and the spatial derivatives carried out using the second-order central difference, and the coupling is enforced by exchanging local fields between these algorithms at each time step. In our simulation, the lattice spacing, time step, and fluid density are all set to unity. The length scale is calibrated based on the length of an active unit approximately $1 \mu \mathrm{m}$ so that the simulation length unit corresponds to $1 \mu \mathrm{m}$ in physical unit. The force scale is set to $10^{-11} \mathrm{~N}$, and the timescale calibration is obtained by assuming the rotational viscosity to be approximately $0.1 \mathrm{~Pa}$ $\mathrm{s}$; therefore, the simulation time unit equates to $1 \mathrm{~ms}$. $\tau^{*}$ corresponds to $10^{3}$ simulation time steps. For $|r| \leq 200$, where $r$ is the position vector of the lattice point with respect to the center of particle, the system is initialized with the director field aligned tangent to a series of concentric circles centered around the particle's centroid (inner region). Outside this region, the director has a random orientation. The thickness of this effective hydrodynamic length could be tuned by the particle angular velocity, the elasticity of the liquid-crystal medium, and also the activity strength. In our simulation, we assume that the system consists of elongated self-driven units (active particles) which have long-range orientational ordering. The system is maintained out of equilibrium by the forces that these active units exert on their surrounding environment. We perform our simulation by adding the active stress of an extensile force dipole to the system, and this energy input results in the continuous generation of defects. We further assume that the bacterial concentration is uniform and neglect its concentration fluctuations and also the active curvature current. In our 2D simulation, we assume that the loss of momentum to the surrounding is negligible, so the momentum is conserved and the active unit energy is not damped by the frictional forces. Before adding the extensile active stress to the system, we let the director within the outer region dynamically evolve and form a large number of defects while keeping the directors within the inner region fixed to impede them from evolution. This setup mimics hydrodynamic flow alignment of liquid crystals due to the rotation of a colloid. We then remove this restriction and let the whole system dynamically evolve. Upon releasing this restriction, the undulation of the director field accompanied with a large bending deformation emerges. These activity-induced instabilities grow radially toward the particle.

\section{APPENDIX C: EMERGENCE OF TOPOLOGICAL DEFECTS}

Undulations of the director field and the transition to chaotic motion lead to a consequent creation of $+1 / 2$ and $-1 / 2$ pairs of topological defects. To capture this process, we process a series of images recorded immediately after cessation of the rotation. Defects are detected by processing a director field and finding nonzero topological charges. The emergence of topological defects as obtained in experiments and simulations is shown in Fig. 4.

\section{APPENDIX D: BACTERIAL DENSITY VARIATION}

To estimate variations of the local bacterial concentration in our experiments, we use a custom-made MATLAB script based on an edge detection algorithm. We process several 


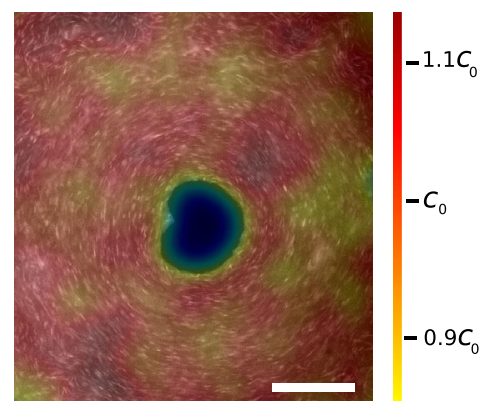

(a)

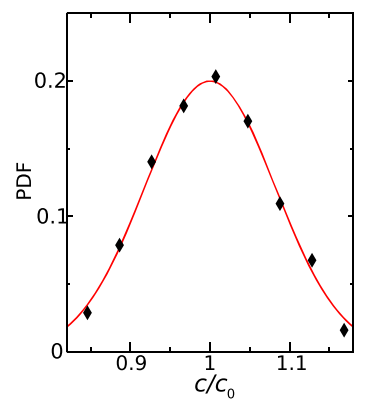

(b)
FIG. 9. (a) Example of a bacterial density distribution immediately after cessation of rotation. The average bacterial concentration $c_{0} \sim 10^{9}$ cell per $\mathrm{cm}^{3}$. Red corresponds to a higher bacterial concentration. The scale bar is $60 \mu \mathrm{m}$. (b) Probability distribution function for local bacterial concentrations over the field of view shown in (a). Standard deviation $\sigma \approx 0.08$.

images of circularly aligned fluorescent bacteria near the bottom surface of the drop immediately after the cessation of rotation. The number of bacteria is assumed to be proportional to the perimeter of detected bacterial edges. The accuracy of the algorithm is tested manually for small areas $(10 \mu \mathrm{m} \times 10 \mu \mathrm{m})$. The variation of bacterial concentration in a typical experiment is less than 10\%; see Fig. 9. After the development of instability and transition to a chaotic motion, such variations increase due to the trapping of bacteria by topological defects [29].

[1] M. C. Marchetti, J.-F. Joanny, S. Ramaswamy, T. B. Liverpool, J. Prost, M. Rao, and R. A. Simha, Hydrodynamics of Soft Active Matter, Rev. Mod. Phys. 85, 1143 (2013).

[2] P. G. de Gennes and J. Prost, The Physics of Liquid Crystals (Oxford University, New York, 1995).

[3] M. J. Stephen and J. P. Straley, Physics of Liquid Crystals, Rev. Mod. Phys. 46, 617 (1974).

[4] P. M. Chaikin, T. C. Lubensky, and T. A. Witten, Principles of Condensed Matter Physics (Cambridge University Press, Cambridge, England, 1995), Vol. 1.

[5] H. Stark, Physics of Colloidal Dispersions in Nematic Liquid Crystals, Phys. Rep. 351, 387 (2001).

[6] M. Kleman and O. D. Laverntovich, Soft Matter Physics: An Introduction (Springer Science, New York, 2007).

[7] A. D. Rey and M. M. Denn, Dynamical Phenomena in Liquid-Crystalline Materials, Annu. Rev. Fluid Mech. 34, 233 (2002).

[8] R. G. Larson, The Structure and Rheology of Complex Fluids (Oxford University Press, New York, 1999).

[9] J. Han, Y. Luo, W. Wang, P. Zhang, and Z. Zhang, From Microscopic Theory to Macroscopic Theory: A Systematic Study on Modeling for Liquid Crystals, Arch. Ration. Mech. Anal. 215, 741 (2015).

[10] A. N. Beris and B. J. Edwards, Thermodynamics of Flowing Systems: With Internal Microstructure (Oxford University, New York, 1994).
[11] O. D. Lavrentovich, Transport of Particles in Liquid Crystals, Soft Matter 10, 1264 (2014).

[12] A. Doostmohammadi, J. Ignés-Mullol, J. M. Yeomans, and F. Sagués, Active Nematics, Nat. Commun. 9, 3246 (2018).

[13] T. Sanchez, D. T. N. Chen, S. J. DeCamp, M. Heymann, and Z. Dogic, Spontaneous Motion in Hierarchically Assembled Active Matter, Nature (London) 491, 431 (2012).

[14] G. Henkin, S. J. DeCamp, D. T. N. Chen, T. Sanchez, and Z. Dogic, Tunable Dynamics of Microtubule-Based Active Isotropic Gels, Phil. Trans. R. Soc. A 372, 20140142 (2014).

[15] G. Vliegenthart, A. Ravichandran, M. Ripoll, T. Auth, and G. Gompper, Filamentous Active Matter: Band Formation, Bending, Buckling, and Defects, arXiv:1902.07904.

[16] V. Schaller, C. Weber, C. Semmrich, E. Frey, and A. R. Bausch, Polar Patterns of Driven Filaments, Nature (London) 467, 73 (2010).

[17] N. Kumar, R. Zhang, J. J. de Pablo, and M. L. Gardel, Tunable Structure and Dynamics of Active Liquid Crystals, Sci. Adv. 4, eaat7779 (2018).

[18] R. Zhang, N. Kumar, J. Ross, M. L. Gardel, and J. J. de Pablo, Interplay of Structure, Elasticity, and Dynamics in Actin-Based Nematic Materials, Proc. Natl. Acad. Sci. U.S.A. 115, E124 (2018).

[19] C. Dombrowski, L. Cisneros, S. Chatkaew, R. E. Goldstein, and J. O. Kessler, Self-Concentration and Large-Scale Coherence in Bacterial Dynamics, Phys. Rev. Lett. 93, 098103 (2004).

[20] H. Li, X.-q. Shi, M. Huang, X. Chen, M. Xiao, C. Liu, H. Chaté, and H. P. Zhang, Data-Driven Quantitative Modeling of Bacterial Active Nematics, Proc. Natl. Acad. Sci. U.S.A. 116, 777 (2019).

[21] G. Duclos, S. Garcia, H. G. Yevick, and P. Silberzan, Perfect Nematic Order in Confined Monolayers of Spindle-Shaped Cells, Soft Matter 10, 2346 (2014).

[22] R. Mueller, J. M. Yeomans, and A. Doostmohammadi, Emergence of Active Nematic Behavior in Monolayers of Isotropic Cells, Phys. Rev. Lett. 122, 048004 (2019).

[23] G. Duclos, C. Erlenkämper, J.-F. Joanny, and P. Silberzan, Topological Defects in Confined Populations of SpindleShaped Cells, Nat. Phys. 13, 58 (2017).

[24] A. Doostmohammadi, S. P. Thampi, and J. M. Yeomans, Defect-Mediated Morphologies in Growing Cell Colonies, Phys. Rev. Lett. 117, 048102 (2016).

[25] T. B. Saw, A. Doostmohammadi, V. Nier, L. Kocgozlu, S. Thampi, Y. Toyama, P. Marcq, C. T. Lim, J. M. Yeomans, and B. Ladoux, Topological Defects in Epithelia Govern Cell Death and Extrusion, Nature (London) 544, 212 (2017).

[26] K. Kawaguchi, R. Kageyama, and M. Sano, Topological Defects Control Collective Dynamics in Neural Progenitor Cell Cultures, Nature (London) 545, 327 (2017).

[27] S. Zhou, A. Sokolov, O. D. Lavrentovich, and I. S. Aranson, Living Liquid Crystals, Proc. Natl. Acad. Sci. U. S. A. 111, 1265 (2014).

[28] C. Peng, T. Turiv, Y. Guo, Q.-H. Wei, and O. D. Lavrentovich, Command of Active Matter by Topological Defects and Patterns, Science 354, 882 (2016).

[29] M. M. Genkin, A. Sokolov, O. D. Lavrentovich, and I. S. Aranson, Topological Defects in a Living Nematic Ensnare Swimming Bacteria, Phys. Rev. X 7, 011029 (2017). 
[30] M. M. Genkin, A. Sokolov, and I. S. Aranson, Spontaneous Topological Charging of Tactoids in a Living Nematic, New J. Phys. 20, 043027 (2018).

[31] R. A. Simha and S. Ramaswamy, Hydrodynamic Fluctuations and Instabilities in Ordered Suspensions of SelfPropelled Particles, Phys. Rev. Lett. 89, 058101 (2002).

[32] L. H. Cisneros, J. O. Kessler, S. Ganguly, and R. E. Goldstein, Dynamics of Swimming Bacteria: Transition to Directional Order at High Concentration, Phys. Rev. E 83, 061907 (2011).

[33] L. Giomi, Geometry and Topology of Turbulence in Active Nematics, Phys. Rev. X 5, 031003 (2015).

[34] B. Martínez-Prat, J. Ignés-Mullol, J. Casademunt, and F. Sagués, Selection Mechanism at the Onset of Active Turbulence, Nat. Phys. 15, 362 (2019).

[35] L. Giomi, M. J. Bowick, X. Ma, and M. C. Marchetti, Defect Annihilation and Proliferation in Active Nematics, Phys. Rev. Lett. 110, 228101 (2013).

[36] L. Giomi, M. J. Bowick, P. Mishra, R. Sknepnek, and M. C. Marchetti, Defect Dynamics in Active Nematics, Phil. Trans. R. Soc. A 372, 20130365 (2014).

[37] S. J. DeCamp, G. S. Redner, A. Baskaran, M. F. Hagan, and Z. Dogic, Orientational Order of Motile Defects in Active Nematics, Nat. Mater. 14, 1110 (2015).

[38] A. Doostmohammadi, M. F. Adamer, S. P. Thampi, and J. M. Yeomans, Stabilization of Active Matter by FlowVortex Lattices and Defect Ordering, Nat. Commun. 7, 10557 (2016).

[39] A. U. Oza and J. Dunkel, Antipolar Ordering of Topological Defects in Active Liquid Crystals, New J. Phys. 18, 093006 (2016).

[40] P. W. Ellis, D. J. G. Pearce, Y.-W. Chang, G. Goldsztein, L. Giomi, and A. Fernandez-Nieves, Curvature-Induced Defect Unbinding and Dynamics in Active Nematic Toroids, Nat. Phys. 14, 85 (2018).

[41] P. Guillamat, Ž. Kos, J. Hardoüin, J. Ignés-Mullol, M. Ravnik, and F. Sagués, Active Nematic Emulsions, Sci. Adv. 4, eaa01470 (2018).

[42] Suraj Shankar, Sriram Ramaswamy, M Cristina Marchetti, Mark J Bowick, Defect Unbinding in Active Nematics, Phys. Rev. Lett. 121, 108002 (2018).

[43] A. Joshi, E. Putzig, A. Baskaran, and M. F. Hagan, The Interplay between Activity and Filament Flexibility Determines the Emergent Properties of Active Nematics, Soft Matter 15, 94 (2019).

[44] A. Opathalage, M. M. Norton, M. P. N. Juniper, B. Langeslay, S. A. Aghvami, S. Fraden, and Z. Dogic, Self-Organized Dynamics and the Transition to Turbulence of Confined Active Nematics, Proc. Natl. Acad. Sci. U.S.A. 116, 4788 (2019).

[45] T. Gao, M. D. Betterton, A.-S. Jhang, and M. J. Shelley, Analytical Structure, Dynamics, and Coarse Graining of a Kinetic Model of an Active Fluid, Phys. Rev. Fluids 2, 093302 (2017).

[46] M. Ravnik and J. M. Yeomans, Confined Active Nematic Flow in Cylindrical Capillaries, Phys. Rev. Lett. 110, 026001 (2013).

[47] E. Lushi, H. Wioland, and R. E. Goldstein, Fluid Flows Created by Swimming Bacteria Drive Self-Organization in
Confined Suspensions, Proc. Natl. Acad. Sci. U.S.A. 111, 9733 (2014).

[48] J. Hardoüin, R. Hughes, A. Doostmohammadi, J. Laurent, T. Lopez-Leon, J. M. Yeomans, J. Ignés-Mullol, and F. Sagués, Reconfigurable Flows and Defect Landscape of Confined Active Nematics, arXiv:1903.01787

[49] T. N. Shendruk, A. Doostmohammadi, K. Thijssen, and J. M. Yeomans, Dancing Disclinations in Confined Active Nematics, Soft Matter 13, 3853 (2017).

[50] A. Doostmohammadi, T. N. Shendruk, K. Thijssen, and J. M. Yeomans, Onset of Meso-Scale Turbulence in Active Nematics, Nat. Commun. 8, 15326 (2017).

[51] G. Duclos, C. Blanch-Mercader, V. Yashunsky, G. Salbreux, J.-F. Joanny, J. Prost, and P. Silberzan, Spontaneous Shear Flow in Confined Cellular Nematics, Nat. Phys. 14, 728 (2018).

[52] K.-T. Wu, J. Bernard Hishamunda, D. T. N. Chen, S. J. DeCamp, Y.-W. Chang, A. Fernández-Nieves, S. Fraden, and Z. Dogic, Transition from Turbulent to Coherent Flows in Confined Three-Dimensional Active Fluids, Science 355, eaal1979 (2017).

[53] L. Giomi, L. Mahadevan, B. Chakraborty, and M. F. Hagan, Excitable Patterns in Active Nematics, Phys. Rev. Lett. 106, 218101 (2011).

[54] S. P. Thampi, R. Golestanian, and J. M. Yeomans, Instabilities and Topological Defects in Active Nematics, Europhys. Lett. 105, 18001 (2014).

[55] P. Srivastava, P. Mishra, and M. C. Marchetti, Negative Stiffness and Modulated States in Active Nematics, Soft Matter 12, 8214 (2016).

[56] S. P. Thampi and J. M. Yeomans, Active Turbulence in Active Nematics, Eur. Phys. J. Spec. Top. 225, 651 (2016).

[57] S. P. Thampi, A. Doostmohammadi, T. N. Shendruk, R. Golestanian, and J.M. Yeomans, Active Micromachines: Microfluidics Powered by Mesoscale Turbulence, Sci. Adv. 2, e1501854 (2016).

[58] H. Wioland, F. G. Woodhouse, J. Dunkel, and R. E. Goldstein, Ferromagnetic and Antiferromagnetic Order in Bacterial Vortex Lattices, Nat. Phys. 12, 341 (2016).

[59] D. Nishiguchi, I. S. Aranson, A. Snezhko, and A. Sokolov, Engineering Bacterial Vortex Lattice via Direct Laser Lithography, Nat. Commun. 9, 4486 (2018).

[60] M. L. Blow, M. Aqil, B. Liebchen, and D. Marenduzzo, Motility of Active Nematic Films Driven by Active Anchoring, Soft Matter 13, 6137 (2017).

[61] M. M. Norton, A. Baskaran, A. Opathalage, B. Langeslay, S. Fraden, A. Baskaran, and M. F. Hagan, Insensitivity of Active Nematic Liquid Crystal Dynamics to Topological Constraints, Phys. Rev. E 97, 012702 (2018).

[62] See Supplemental Material at http://link.aps.org/ supplemental/10.1103/PhysRevX.9.031014 for Supplemental Videos.

[63] A. Sokolov and I.S. Aranson, Rapid Expulsion of Microswimmers by a Vortical Flow, Nat. Commun. 7, 11114 (2016).

[64] A. Sokolov, L. D. Rubio, J. F. Brady, and I. S. Aranson, Instability of Expanding Bacterial Droplets, Nat. Commun. 9, 1322 (2018).

[65] A. Sokolov, S. Zhou, O. D. Lavrentovich, and I. S. Aranson, Individual Behavior and Pairwise Interactions between 
Microswimmers in Anisotropic Liquid, Phys. Rev. E 91, 013009 (2015).

[66] D. Marenduzzo, E. Orlandini, and J. M. Yeomans, Hydrodynamics and Rheology of Active Liquid Crystals: A Numerical Investigation, Phys. Rev. Lett. 98, 118102 (2007).

[67] D. Marenduzzo, E. Orlandini, M. E. Cates, and J. M. Yeomans, Steady-State Hydrodynamic Instabilities of Active Liquid Crystals: Hybrid Lattice Boltzmann Simulations, Phys. Rev. E 76, 031921 (2007).

[68] R. Voituriez, J.-F. Joanny, and J. Prost, Spontaneous Flow Transition in Active Polar Gels, Europhys. Lett. 70, 404 (2005).

[69] S. A. Edwards and J. M. Yeomans, Spontaneous Flow States in Active Nematics: A Unified Picture, Europhys. Lett. 85, 18008 (2009).
[70] K. M. Blair, L. Turner, J. T. Winkelman, H. C. Berg, and D. B. Kearns, A Molecular Clutch Disables Flagella in the Bacillus Subtilis Biofilm, Science 320, 1636 (2008).

[71] S. Zhou, Elasticity, Viscosity, and Orientational Fluctuations of a Lyotropic Chromonic Nematic Liquid Crystal Disodium Cromoglycate, in Lyotropic Chromonic Liquid Crystals (Springer, New York, 2017), pp. 51-75.

[72] A. Sokolov, I.S. Aranson, J. O. Kessler, and R. E. Goldstein, Concentration Dependence of the Collective Dynamics of Swimming Bacteria, Phys. Rev. Lett. 98, 158102 (2007).

[73] J. B. Fournier and P. Galatola, Modeling Planar Degenerate Wetting and Anchoring in Nematic Liquid Crystals, Europhys. Lett. 72, 403 (2005). 